\title{
Bending induced self-organized reversible gratings on polymeric substrates
}

Julian Parra-Barranco ${ }^{1}$, Manuel Oliva-Ramirez ${ }^{1}$, Lola Gonzalez-Garcia ${ }^{1}$, Maria Alcaire ${ }^{1}$, Manuel Macias-Montero ${ }^{1}$, Ana Borras ${ }^{1}$, Fabian Frutos ${ }^{2}$, Agustin R. Gonzalez-Elipe ${ }^{1}$, Angel Barranco $^{1 *}$

${ }^{1}$ Consejo Superior de Investigaciones Científicas. Instituto de Ciencia de Materiales de Sevilla (CSIC-Universidad de Sevilla). c/ Américo Vespucio 49, ES 41092 Sevilla, Spain.

${ }^{2}$ Dept. Física Aplicada, E.T.S. Ingeniería Informática, Universidad de Sevilla, Avda. Reina Mercedes s/n, ES 41012 Sevilla, Spain.

*Corresponding author e-mail: angel.barranco@csic.es

After prolonged immersion in water the skin expands producing the universally experienced pruney fingers. Similar surface instabilities are ubiquitous in nature and arise whenever a stiff film coats a compliant substrate. The field of surface instabilities in engineered thin films is in continuous expansion since the pioneered publications by Whiteshides and co-workers on hierarchical surface patterning ${ }^{1-3}$ that have inspired a rich variety of applications ${ }^{4-10}$. Herein we propose a straightforward procedure of self-surface patterning with potential applications as large area gratings, invisible labeling, optomechanical transducers or smart windows. The methodology is based in the formation of parallel micrometric crack patterns when polydimethylsiloxane (PDMS) foils coated with tilted nanocolumnar $\mathrm{SiO}_{2}$ thin films are manually bent. The crack spacing is controlled by the film nanostructure and is independent of the film thickness and bending curvature. These self-organized patterned foils are completely transparent and work as customized reversible diffraction gratings under mechanical activation.

In general, cracking of thin films is synonym of irreversible damage, delamination and/or device failure. Nevertheless, under certain well controlled conditions, a tensile stress applied to a supported film can induce the development of a regular pattern perpendicular to the resulting strain $^{11,12}$. For example, patterns of cracks produced by uniaxial straining of plasma surface 
oxidized PDMS foils have been used for the fabrication of protein matrices ${ }^{13}$ and nanofluidic channels ${ }^{14}$. A simpler way of applying stress to a PDMS foil is by bending. We have found that when this elastomeric polymer coated with a relatively rigid silicon dioxide layer is bent, irregular cracks are formed to release the accumulated mechanical stress. Fig.S1 a-c in the Supplementary Information shows a series of micrographs of PDMS foils bent after being coated with continuous $\mathrm{SiO}_{2}$ thin films. These experiments have been conducted with $\mathrm{SiO}_{2} / \mathrm{PDMS}$ systems fabricated by surface oxidation with an oxygen plasma ${ }^{15 a}$ (cf. S1a), plasma-enhanced chemical vapor deposition ${ }^{15 b}$ (cf. S1b) or electron evaporation of silica at normal incidence (cf. S1c), respectively. In the three cases, bending slightly the coated foils produced a series of irregular cracks whose number increases with additional bending operations. This accumulated cracking is neither controllable nor reproducible and typically yields local delamination after a small number of bending events. By contrast, bending a PDMS foil coated with a nanocolumnar $\mathrm{SiO}_{2}$ thin film prepared by physical vapor deposition at glancing angles (GLAD) renders quite different results. This deposition technique produces anisotropic porous thin films characterized by a tilted columnars microstructure resulting from shadowing effects during the growth process ${ }^{16-18}$. The nanocolumns tilting angle and porosity of these films increase gradually with the deposition angle while, simultaneously, the number of nanocolumns per unit area decreases ${ }^{19,20}$. In some materials like $\mathrm{SiO}_{2}$ the tilted nanocolumns tend to aggregate in the form of parallel nanochannels arranged perpendicular to the direction of the incoming flux. This process, known as bundling ${ }^{22}$ has been recently exploited for the fabrication of tunable dichroic optical structures ${ }^{23-25}$. As shown in the Supplementary Information $\mathrm{S} 2$, room temperature GLAD deposition of $\mathrm{SiO}_{2}$ on PDMS foils (hereafter called $\mathrm{G}_{-} \mathrm{SiO}_{2} / \mathrm{PDMS}$ ) yields nanocolumnar thin films where the bundling of the nanocolumns enhances the film in-plane anisotropy ${ }^{23,24}$. 


\section{a)}
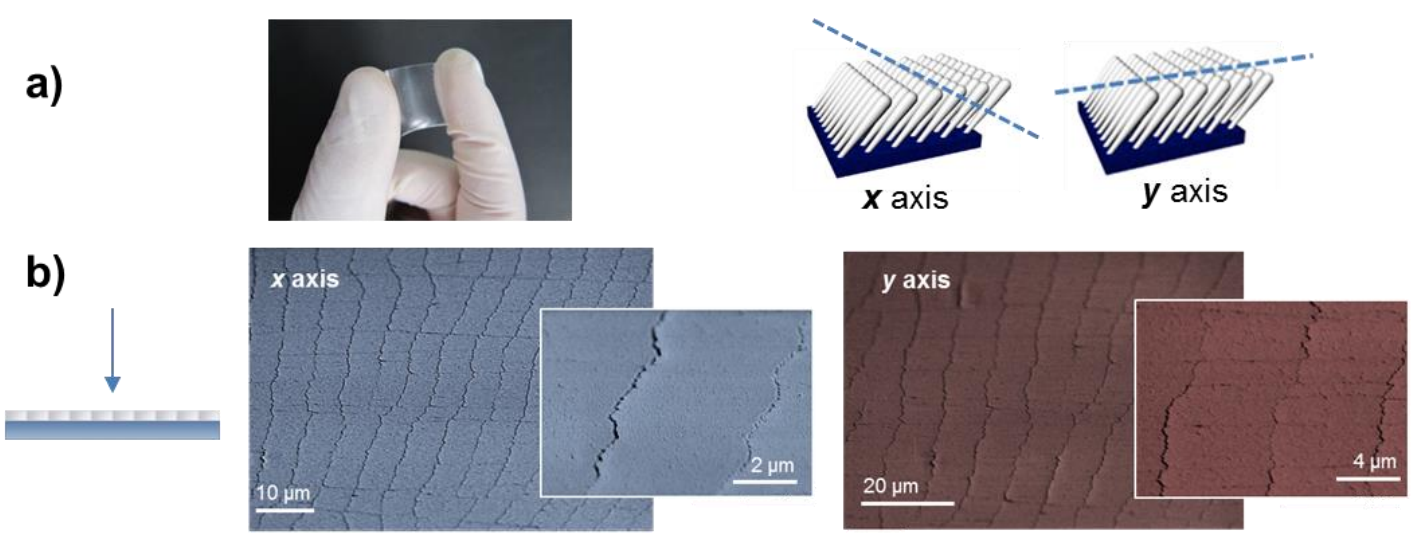

c)
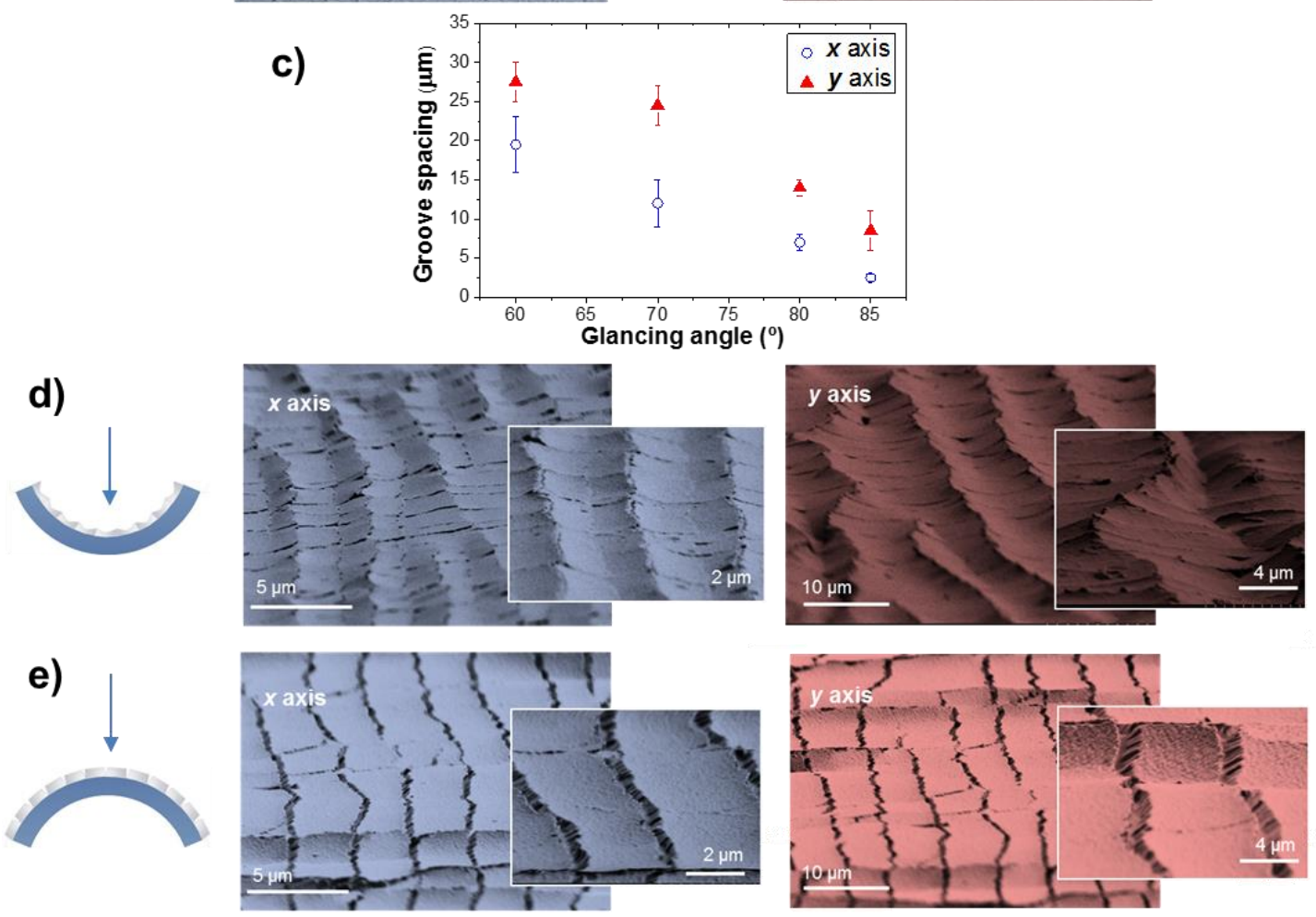

Figure 1 Regular groove formation by bending. a, Picture showing the extremely simple procedure required for structuring the surface of a PDMS foil coated with a $\mathrm{G}_{-} \mathrm{SiO}_{2}$ thin film. The schemes show the two bending direction axis as defined according to the thin film anisotropic structure. A first bending event produces a periodic crack pattern in the nanocolumnar oxide surface that is retained after successive bending events. b, FESEM micrographs of two deposited $\mathrm{G}_{-} \mathrm{SiO}_{2}$ nanocolumnar films after bending the PDMS along the $\boldsymbol{x}$ and $\boldsymbol{y}$ directions. $\mathbf{c}$, Relationship between groove spacing and thin film nanostructure as determined by the angle of evaporation for the films bent along the $x$ and $y$ axes. d, FESEM micrographs of the films in $\mathbf{b}$ forming a concave surface. e, FESEM micrographs of the films in $\mathbf{b}$ forming a convex surface. The tilted nanocolumnar structure of the $\mathrm{G}_{-} \mathrm{SiO}_{2}$ films is clearly appreciable in the high magnification insets of this figure. The pictures and graphs in this figure correspond to a $\sim 300$ $\mathrm{nm}$ film deposited at a glancing angle of $85^{\circ}\left(\mathrm{G}^{\circ}-5^{\circ} \mathrm{SiO}_{2}\right)$. 
After thin film deposition, the $\mathrm{G}-\mathrm{SiO}_{2} / \mathrm{PDMS}$ foils were bent as shown in Fig. 1a along the orthogonal $\boldsymbol{y}$ and $\boldsymbol{x}$ axis defined by the nanocolumnar growth and bundling directions, respectively. Fig. $1 b$ shows that bending along the $x$ axis a $\mathrm{G}^{\circ} 5^{\circ}-\mathrm{SiO}_{2} / \mathrm{PDMS}$ foil renders a set of parallel and homogeneously separated grooves that, from edge to edge, cover the whole film surface. These grooves, spaced by $\sim 4 \mu \mathrm{m}$, result from a regular cracking of the deposited oxide and define a regular pattern parallel to the bending axis that remains unaltered after bending the film for more than one thousand times (literally as will be shown below). Bending the same foil along the $\boldsymbol{y}$ axis yields a similar pattern with a spacing between grooves of $\sim 6 \mu \mathrm{m}$. It could be realized that the inter-groove spacing could be greatly varied by just changing the deposition angle of the $\mathrm{G}_{-} \mathrm{SiO}_{2}$ films (c.f. Figure 1c) and that neither the film thickness nor the bending curvature had any appreciable influence on the pattern characteristics.

Further insight into the dynamic of the grooves upon successive bending was gained by keeping curved the $\mathrm{G}-\mathrm{SiO}_{2} / \mathrm{PDMS}$ foils during SEM observation. The micrographs in Fig. 1d for the film situated onto the concave side of the foil curved along the $x$ or $y$ bending axes show that the accumulated compressive stress releases through the formation of wrinkles consisting of a periodic distribution of valleys and ridges. This wrinkles arrangement is a direct transformation of the parallel groove pattern observed on the flat surfaces, with the wrinkling period and spacing coinciding with those of the initial cracks. By contrast, the normal view micrographs of the films placed on the convex side (Fig. 1e) show the development of similarly spaced big grooves where the gap separating the cracked $\mathrm{SiO}_{2}$ strips is larger than in the flat state.

A remarkable property of the groove structure generated by bending the $\mathrm{G}-\mathrm{SiO}_{2} / \mathrm{PDMS}$ surfaces is that they work as reversible optical gratings. Figure 2a shows that the flat or bent foils with the $\mathrm{G}_{-} \mathrm{SiO}_{2}$ film at the convex side were transparent. By contrast, in the right side photograph in this figure, when the bent foil exposes the $\mathrm{G}-\mathrm{SiO}_{2}$ film at the concave side, the foil surface wrinkled nanostructure acts as a diffraction grating splitting a white light beam into its components. Diffraction is further proved by the inset showing the formation well-defined first order spectral images at both sides of an illuminated cut-out figure observed through the 
concave $\mathrm{G}-\mathrm{SiO}_{2} / \mathrm{PDMS}$ foil. This transition from a transparent configuration (i.e., flat or convex surface) to a grating configuration was fully reversible and reproducible by repeating the process more than one thousand times with the same foil. An outstanding characteristic of the diffracted patterns was that the positions and intensities of diffracted lines did not depend on the surface curvature but on the deposition angle of the films and, therefore, they were entirely controllable by the manufacturing process. In all cases a minimal curvature of the surface was sufficient to induce the diffraction of light.

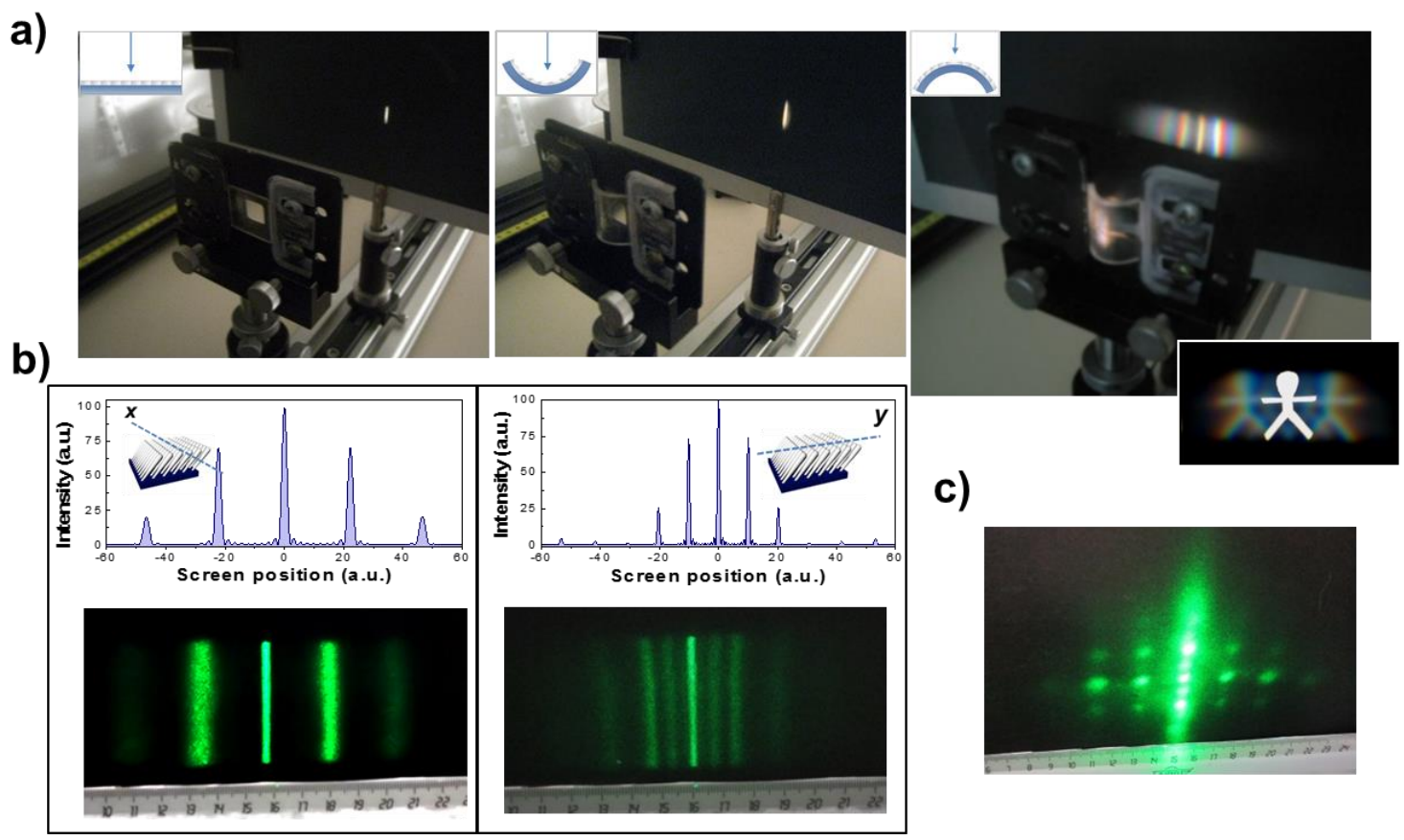

Figure 2 Reversible grating effects. a, Pictures of a white light beam passing through a G-SiO $2-\mathrm{PDMS}$ foil with a regular groove pattern formed by bending. The light passing through this foil in a flat (left) and convex (center) configuration reaches the screen undisturbed. The light passing through the concave covered surface (right) is diffracted and forms a characteristic rainbow pattern onto the screen. The inset in the right image corresponds to a white light back-illuminated cut-out figure observed through a concave $\mathrm{G}-\mathrm{SiO}_{2} / \mathrm{PDMS}$ foil. b, Diffraction patterns obtained with two concave surfaces of the same G$\mathrm{SiO}_{2} / \mathrm{PDMS}$ foil illuminated with a $532 \mathrm{~nm}$ laser focused along a line parallel to the $\boldsymbol{x}$ and $\boldsymbol{y}$ bending axis. The calculated Fraunhofer diffraction patterns for the two axes are also included in the figure. c, Diffraction of a $532 \mathrm{~nm}$ laser dot by a G-SiO $2 / \mathrm{PDMS}$ foil bent by pressing the four corners simultaneously with the oxide film covering the concave hemispherical surface. All the examples of this figure correspond to a $\sim 300 \mathrm{~nm}$ thick $\mathrm{G} 85^{\circ} \mathrm{SiO}_{2} / \mathrm{PDMS}$ foil. 
To understand the origin of these diffraction effects we illuminated two concave surfaces with a $532 \mathrm{~nm}$ laser focused in a line oriented either along the $\boldsymbol{x}$ or $\boldsymbol{y}$ bending axes (Figure 2b) and measured the distance between the diffraction maxima and the relative intensity of the individual lines. The experimental patterns can be directly simulated (Figure $2 \mathrm{~b}$ ) by assuming a Fraunhofer diffraction by the periodic undulation observed in the concave bent surfaces (see Fig. 1d and $\mathrm{S} 3$ for details). The optical grating possibilities of the $\mathrm{G}-\mathrm{SiO}_{2} / \mathrm{PDMS}$ foils are further evidenced in Fig. $2 d$ showing the diffraction pattern obtained when the concave surface adopts a hemispherical shape by pressing simultaneously the four corners of the foil. In this case, the laser diffraction pattern is a combination of the single patterns obtained by edge bending along the $\boldsymbol{x}$ and $\boldsymbol{y}$ axis. A similar pattern was obtained when the foils were bent along an axis different to $\boldsymbol{x}$ or $\boldsymbol{y}$. These evidences support that the $\boldsymbol{x}$ and $\boldsymbol{y}$ axes are determined by specific directions of the $\mathrm{G}_{-} \mathrm{SiO}_{2}$ film nanostructure and not by the foil manipulation.

To elucidate the relationship between thin film microstructure and surface patterning upon bending, a set of $\mathrm{G}-\mathrm{SiO}_{2} / \mathrm{PDMS}$ foils with different oxide thicknesses have been prepared at different glancing angles of deposition. Fig. 1c shows that the groove spacing systematically decreases with the evaporation angle and is always higher by bending along the $\boldsymbol{y}$ axis (see also the Supplementary Information S3 for additional examples). Similar experiments carried out with $\mathrm{G}_{-} \mathrm{TiO}_{2} / \mathrm{PDMS}$ foils showed a similar decreasing trend, although in this system no significant differences were found by bending along the $\boldsymbol{x}$ or $\boldsymbol{y}$ axis (see S5). This different behavior can be linked with the low tendency of the $\mathrm{TiO}_{2}$-GLAD films to develop bundles of nanocolumns ${ }^{19,20}$ and stresses the importance of the film nanostructure for the control of the micropatterning processes. Another interesting feature of the $\mathrm{G}_{-} \mathrm{SiO}_{2} / \mathrm{PDMS}$ system is that the average crack spacing is independent of the thin film thickness. This was proved by verifying that identical micropatterns are obtained for thin films of $\sim 50,100,300$ and $600 \mathrm{~nm}$ when deposited at the same glancing angle. In the curse of these studies it was also found that groove spacing was independent on the bending magnitude initially applied to the foil to produce the surface cracking. This behavior contrasts with the reported behavior of wrinkled PDMS surfaces 
where wrinkling-related diffraction effects are tightly dependent on the thickness of the stiff films coating the PDMS and on the magnitude of the experienced strain ${ }^{5-7,26,27}$. Other studies about cracking of thin films on compliant substrates also reveal that the average inter-crack distance is always inversely proportional to the strain ${ }^{11-14}$. We attribute the singular behavior of our system to the coupling between the PDMS surface wrinkling developed to accommodate the bending induced strain when forming a concave surface and the tectonic-like behavior of the inter-crack $\mathrm{G}-\mathrm{SiO}_{2}$ (or $\mathrm{G}-\mathrm{TiO}_{2}$ ) regions that collides forming an specific distribution of valleys and ridges what determines the periodicity of the wrinkles at any curvature (Fig. 1d).

Another remarkable optical effect of the $\mathrm{G}-\mathrm{SiO}_{2} / \mathrm{PDMS}$ foils usable for invisible labeling applications is a global loss of transparency when they are properly bent. Fig. 3a shows that a printed image appears blurred when observed thorough a bent foil with the $\mathrm{G}_{-} \mathrm{SiO}_{2}$ film placed at the convex side. Since the regular groove pattern spreads over the entire bent surface, this effect can be observed through relatively large area foils as in the example of Fig. 3b). In the reported experiment the reduction in transparency was very effective (up to 85\%) and relatively constant along the whole visible spectrum as shown in Fig. 3c. The process was fully reversible and the coated foils became transparent when they were brought back to their flat state (Fig. 3d). In addition, if only a region of the foil is bent, the flat region remains transparent as shown in Fig. 3b). The observed visual loss of transparency was less dependent than the diffraction phenomena on the column microstructure and the specific orientation of the bending axis. A straightforward application of these light dispersion effects is the development of invisible labels, i.e. motives that are invisible when the film is relaxed and visible by bending. Printed figures can be easily designed on the surface of PDMS by depositing the columnar films using shadow masks. An example of this approach is presented in Fig. 3e. The printed structures can be neatly seen on the surface of the bent foil and be projected on a screen by direct white light illumination. Although the fabrication of patterns formed by local surface wrinkling after deposition or plasma oxidation of PDMS foils through shadow masks has been reported previously, ${ }^{2,26}$ a clear advantage of our procedure is that it permits to imprint each side of the 
foil independently. In this way, two different labels can be extracted from a double side coated foil depending on the side forming a convex surface (Figure 3e). Furthermore, if the deposition of a G-SiO 2 film is carried out onto a curved substrate the structure appears either blurred or transparent when the foil is flat or curved, respectively (data not shown).

a)

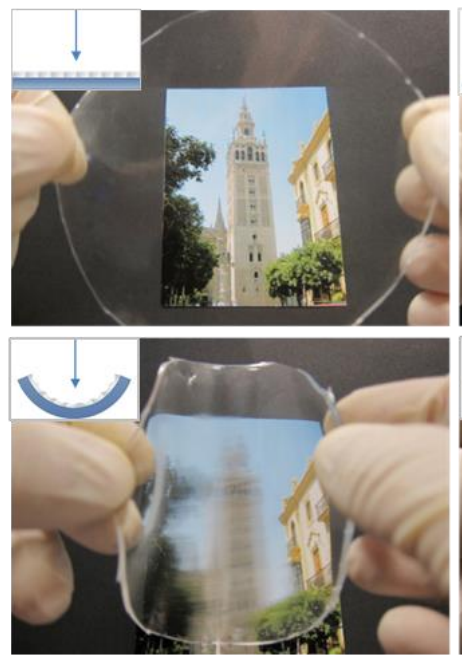

c)

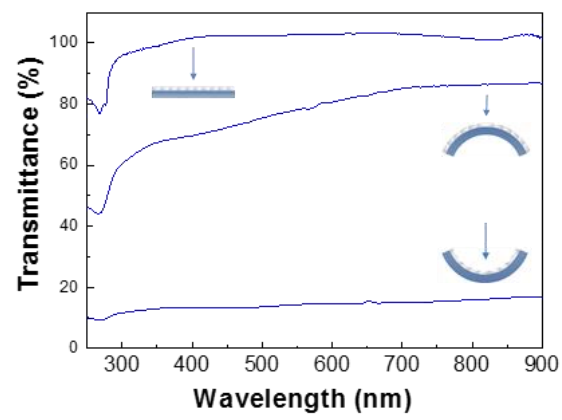

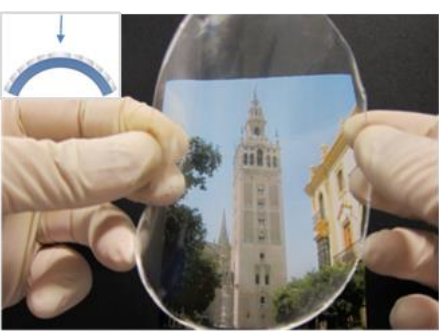

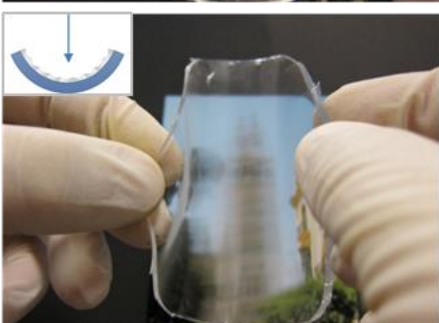

b)

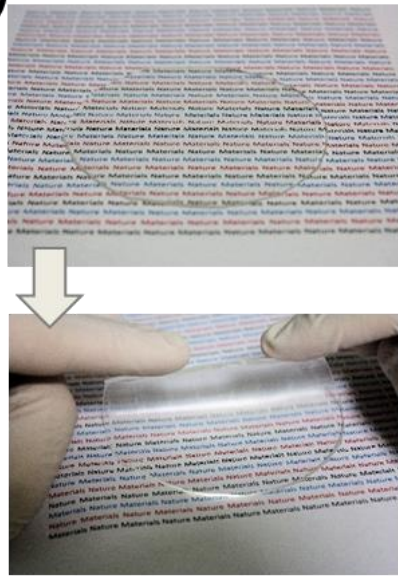

d)

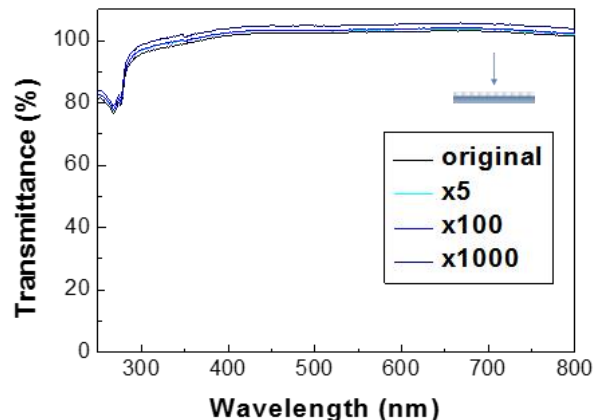

e)

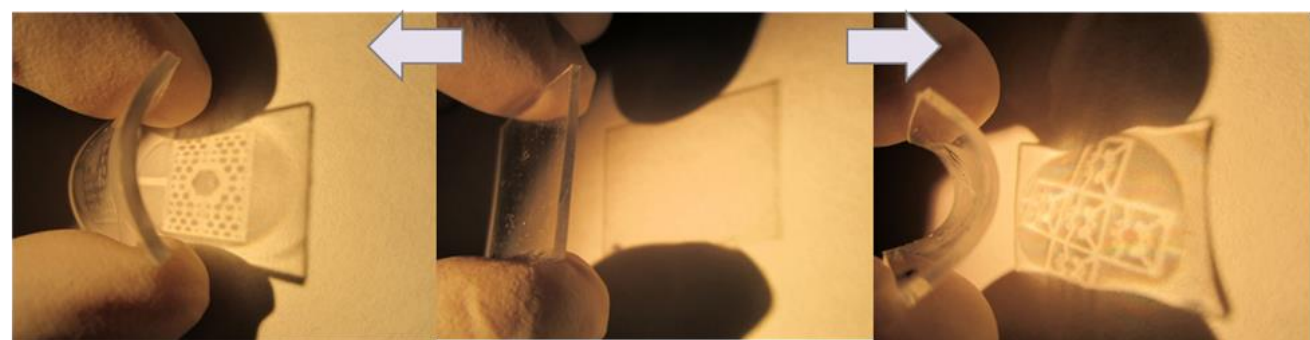

Figure 3 Reversible opacity and invisible labeling. a, Set of pictures demonstrating the loss of transparency of a G-SiO2/PDMS concave surface of a $15 \mathrm{~cm}$ diameter disk in comparison with the normal transparent behavior of the foil in flat and convex configurations. $\mathbf{b}$, the loss of transparency is restricted to the bent zone, whereas the flat region remains transparent. c, UV-Vis transmission spectra corresponding to the flat, convex and concave states as indicated. d, UV-Vis spectra of a $\mathrm{SiO}_{2}$ thin film bent 5, 100 and 1000 times. e, Pictures of a PDMS foil double-side deposited by using two different shadow masks. The projected light reveals the patterns printed onto the convex surface, while for the flat 
and the concave configurations the foil remains transparent. All the examples of this figure correspond to a $\sim 300 \mathrm{~nm}$ thick $\mathrm{SiO}_{2}$ film deposited at $85^{\circ}$ of glancing angle on a PDMS foil.

In this letter we have demonstrated that reversible gratings can be obtained by bending flexible PDMS foils coated with nanocolumnar $\mathrm{SiO}_{2}$ thin films prepared by GLAD. The simplicity of the method is proved by the fact that the PDMS foil, coated at room temperature in a single step, can be bent without any special precaution or consideration about the curvature degree (e.g., any unspecific hand bending produces quite reproducible results). The observation of the surface demonstrates that the patterns, formed by parallel cracks on the flat surface and the associated wrinkles developed on the concave bent surface, are coupled. In comparison with the previously reported patterns formed by wrinkled or cracked films on compliant substrates which are typically obtained by a well-controlled uniaxial straining, the periodic cracking here is induced by a simple bending. Moreover, the magnitude of the externally applied stress (i.e., degree of bending) does not affect the pattern structure which is entirely dependent of the thin film nanostructure. Due to the large variety of single and multilayered nanostructured thin films that can be fabricated by GLAD (i.e., locally anisotropic films, nanostructured multilayers, etc.) and the extreme simplicity of the procedure we can foresee that the methodology outlined here can be a starting point for new micro/nanopatterning design strategies of photonic elements, opto-mechanical actuators, reversible microfluidics valves, controlled wetting or security labeling, among others.

\section{Acknowledgements.}

We thank the Junta de Andalucía (TEP5283) and the Ministry of Economy and Competitiveness (Projects CONSOLIDER-CSD 2008-00023, MAT2010-21228, MAT2010-18447) for financial support.

\section{Materials and methods:}

$\mathrm{SiO}_{2}$ and $\mathrm{TiO}_{2}$ GLAD thin films were prepared by electron evaporation as reported elsewhere (SanchezValencia, Gonzalez-García, Gaillard) at the glancing angles $\theta=60^{\circ}, 70^{\circ}, 80^{\circ}$, and $85^{\circ}$. The temperature of 
the sample holder during the deposition of the $\mathrm{SiO}_{2}$ film was measured to be only 2-4 degrees higher than room temperature. In the case of $\mathrm{TiO}_{2}$ a water cooled sample holder was used to prevent sample heating during deposition. PDMS foils were used as substrates. The PDMS foils were prepared by mixing the Sylgard 184 (DOW) two part silicon elastomer and degassing and curing the mixture at $50^{\circ} \mathrm{C}$ during 1 hour. FESEM characterization was carried out with a Hitachi S5200 microscope and the UV-Vis transmission of the films was measured with a Cary 100 spectrophotometer from Varian.

\section{References}

1. Bowden, N., Brittain, S., Evan,s A. G., Hutchinson, J. W. \& Whitesides, G. M. Spontaneus formation of ordered structures in thin films of metals supported on an elastomeric polymer. Nature 393, 146-149 (1998).

2. Bowden, N., Huck, W. T. S., Paul, K. E. \& Whitesides, G. M. The controlled formation of ordered, sinusoidal structures by plasma oxidation of an elastomeric polymer. Appl. Phys. Lett. 75, 2557-2559 (1998).

3. Huck, W.T.S. et al. Ordering of spontaneously formed buckles on planar surfaces. Langmuir 16, 3497-3501 (2000).

4. Tawfick, S. et al. Engineering of micro- and nanostructured surfaces with anisotropic geometries and properties. Adv. Mater. 24, 1628-1674 (2012).

5. Genzer, J. \& Groenewold, J. Soft matter with hard skin: From skin wrinkles to templating and material characterization. Soft. Mater. 2, 310-323 (2006).

6. Chung, J. Y., Nolte, A. J. \& Stafford, C. Surface wrinkling: A versatile platform for measuring thin-film properties. Adv. Mater. 23, 349-368 (2011).

7. Stafford, C. M. et al. A buckling-based metrology for measuring the elastic moduli of polymeric films. Nat. Mater. 3, 545-550 (2004).

8. Efimenko, K. et al. Nested self-similar wrinkling patterns in skins. Nat. Mater. 4, 293-297 (2005).

9. Maruyama, T., Hirakata, H., Yonezu, A. \& Minoshima, K. Realization of freestanding wrinkled thin films with flexible deformability. Appl. Phys. Lett.98, 041908 (2011).

10. Chung, J. Y., Chastek, T. Q., Fasolka, M. J., Ro H. W. \& Stafford, C. M. Quantifying residual stress in nanoscale thin polymer films via surface wrinkling. ACS Nano 3, 844-852 (2009).

11. Thouless, M. D. Crack spacing in brittle films on an elastic substrate. J. Am. Ceram. Soc. 73 , 2144-2146 (1990).

12. Thouless, M. D., Li, Z., Douville, N. J. \& Takayama, S. Periodic cracking of films supported on compliant substrates. J. Mech. Phys. Solids 59, 1927-1937 (2011). 
13. Zhu, X. et al. Fabrication of reconfigurable protein matrices by cracking. Nat. Mat.4, 403406 (2005).

14. Huh, D. et al. Tunable elastomeric nanochannels for nanofluidic manipulation. Nat. Mater.6, 424-428 (2007).

15. a) Alcaire, M. et al. Soft plasma processing of organic nanowires: a route for the fabrication of 1D organic heterostructures and the template synthesis of inorganic 1D nanostructures. Nanoscale 3, 4554-4559 (2011); b) Barranco, A. et al. Synthesis of $\mathrm{SiO}_{2}$ and $\mathrm{SiO}_{\mathrm{x}} \mathrm{C}_{\mathrm{y}} \mathrm{H}_{\mathrm{z}}$ thin films by microwave plasma CVD, Thin Solid Films 401, 150-158 (2001).

16. Robbie, K. \& Brett, M. J. Sculptured thin films and glancing angle deposition: Growth mechanics and applications. J. Vac. Sci. Technol. A 15, 1460-1467 (1997).

17. Hawkeye, M. M. \& Brett, M. J. Glancing angle deposition: Fabrication, properties, and applications of micro- and nanostructured thin films. J. Vac. Sci. Technol. A 25, 1317-1335. (2007).

18. Kennedy, S. R. \& Brett, M. J. Porous broadband antireflection coating by glancing angle deposition. Appl. Opt.42, 4573-4579 (2003).

19. Gonzalez-Garcia, L. et al. Structure of glancing incidence deposited $\mathrm{TiO}_{2}$ thin Films as revealed by grazing incidence small-angle X-ray scattering. ChemPhysChem 11, 2205-2208 (2010).

20. Gaillard, Y., Rico, V. J., Jimenez-Pique, E. \& Gonzalez-Elipe, A. R. Nanoindentation of $\mathrm{TiO}_{2}$ thin films with different nanostructures. J. Phys. D: Appl. Phys.42, 145305 (2009).

21. Sanchez-Valencia, J.R. et al. Incorporation and thermal evolution of rhodamine $6 \mathrm{G}$ dye molecules adsorbed in porous columnar optical $\mathrm{SiO}_{2}$ thin films. Langmuir 25, 9140-9148 (2009).

22. Van Kranenburg, H. \& Lodder, C. Tailoring growth and local composition by obliqueincidence deposition. A review and new experimental data. Mat. Sci. Eng. R. 11, 295-354 (1994).

23. Sanchez-Valencia, J.R. et al. Tunable In-Plane Optical Anisotropy of Ag Nanoparticles Deposited by DC Sputtering onto $\mathrm{SiO}_{2}$ Nanocolumnar Films. Plasmonics 5, 241-250 (2010).

24. Sanchez-Valencia, J.R. et al. Selective dichroic patterning by nanosecond laser treatment of Ag nanostripes. Adv. Mater. 23, 848-853 (2011).

25. Gonzalez-Garcia, L. et al. Tuning dichroic plasmon resonance modes of gold nanoparticles in optical thin films. Adv. Funct. Mater.23, 1655-1663 (2013).

26. Chua, D. B. H., Ng, H. T. \& Li, S. F. Y. Spontaneous formation of complex and ordered structures on oxygenplasma-treated elastomeric polydimethylsiloxane. Appl. Phys. Lett.76, 721723 (2000).

27. Kim, P. et al. Rational design of mechano-responsive optical materials by fine tuning the evolution of strain-dependent wrinkling patterns. Adv. Opt. Mat. DOI:10.1002/adom.201300034 (2013). 